\title{
Photodiode 1/f noise and other types of less known baseband noises in optical telecommunications devices
}

\author{
O. Llopis ${ }^{1}$, S. Azaizia ${ }^{1}$, K. Saleh ${ }^{1}$, A. Ali Slimane ${ }^{1,2}$, A. Fernandez ${ }^{1,2}$ \\ ${ }^{1}$ CNRS ; LAAS ; Université de Toulouse \\ ${ }^{2}$ UPS ; Université de Toulouse \\ Toulouse, France
}

\begin{abstract}
A technique to measure low frequency noise in illuminated photodiodes is presented, and some $1 / \mathrm{f}$ noise results are given for InGaAs devices. The ability of photodiodes to convert laser noise into RF noise is also discussed, together with other types of $1 / \mathrm{f}$ noise arising directly from the optical fiber, and particularly from scattering phenomena inside the fiber.
\end{abstract}

Keywords-optical links; microwave photonics; photodiodes; 1/f noise; phase noise; Rayleigh scattering noise

\section{INTRODUCTION}

Fiber optics links are today essential components of many analog high quality systems, such as time and frequency distribution [1], optoelectronic oscillators [2] and other microwave photonics applications. In these systems, the radio frequency (RF) signal must be transmitted through optical devices while preserving its high quality, and particularly its spectral purity in case of clock applications. The $1 / \mathrm{f}$ noise of active devices is converted into phase noise through the devices nonlinearities, and degrades this spectral purity. Optoelectronic devices are part of this process, such as any electronic device involved in the link. The impact of laser noise in these systems, including laser $1 / \mathrm{f}$ noise, is today well known, and has been described in many papers $[3,4]$. The possible influence of photodiode noise is on the contrary less known, and has been subject to controversy [ 3 to 6]. Moreover, in case of signal transmission on distances in the range of a few kilometers (or more), other noise sources may become predominant, such as optical scattering effects or noise related to stimulated Brillouin scattering (SBS), if no precautions have been taken against the starting up of this phenomenon.

\section{PHOTODIODE EXCESS NOISE}

The noise measured on an optical link is always a combination of the noise of the emitter (laser + modulator) and the receiver (photodiode + amplifier). The case of high frequency noise in these systems is well described in textbooks [7], and can be summarized by the following equation which computes the carrier to noise ratio (CNR) of a simple link (without output amplifier) from the laser RIN and the photodiode thermal noise and shot noise.

$$
C N R=\frac{P^{2} S^{2}\left(m^{2} / 2\right)}{P^{2} S^{2} R I N+\frac{4 k T}{R}+2 q S P}
$$

$P$ being the received optical power, $S$ the photodiode sensitivity, $m$ the modulation index $(m<1)$, RIN the laser relative intensity noise (in $1 / \mathrm{Hz}$ ), $R$ the photodiode load and $q$ the electron charge. Equation (1) is efficient to compute the noise floor of the link, which is dominated by the laser RIN if the received optical power is high (link without optical losses), which can be dominated by the shot noise at medium received power, and which is dominated by the photodiode thermal noise for very low received optical power.

The case of $1 / \mathrm{f}$ noise is of course more complex, whatever this noise is investigated directly at low frequencies (low frequency analog optical link, used to transmit the data from a sensor, as an example) or if it is converted to higher frequencies, close to the carrier, through system nonlinear elements (case of the optical transmission of spectrally pure signals, such as clocks or frequency references). $1 / \mathrm{f}$ fluctuations can be measured on the laser, for both its frequency and amplitude, and it is supposed that the photodiode generates also $1 / \mathrm{f}$ noise, like any electronic device in which a current flows. When an optical link is measured at low frequency, such a $1 / \mathrm{f}$ spectrum is observed, but the problem is to determine the origin of this noise.

To this purpose, two different experimental set-up have been used, each involving two identical photodiodes (Figure 1). In the first set-up, the two photodiodes behave in the same way, and the cross-spectrum of the two output currents is measured. Because the two photodiodes noise sources are uncorrelated, the photodiode noise disappears after a sufficient average, and only the noise coming from the emitter (the laser) remains. In the second set-up, the two photodiodes generate two currents in the same load which are the opposite of each other. If the system is well balanced (same photodiodes and same power received on the photodiodes), the overall output current is null and the laser amplitude fluctuations are also cancelled. Because the two photodiodes noise sources are uncorrelated, this noise is added in the load, and the noise observed on the FFT analyser is the photodiode noise (multiplied by two). 
Figure 2 depicts the result of such an experiment realized with two Thorlabs FGA-04 InGaAs photodiodes and a Mitsubishi FU-68PDF $1550 \mathrm{~nm}$ telecommunication laser module. The output current noise spectral density is measured while flowing in $\mathrm{R}=470 \Omega$ load, and the two photodiodes are illuminated by the laser, which delivers about $15 \mathrm{~mW}$ optical power. It is clear in this experiment that the output $1 / \mathrm{f}$ noise measured on each photodiode is mainly due to the laser $1 / \mathrm{f}$ amplitude fluctuations (laser 1/f RIN). Only at high frequency offset $(100 \mathrm{kHz})$, the photodiode shot noise changes slightly the output current noise spectrum, as shown by a $2 \mathrm{~dB}$ difference between each channel noise and the cross-spectrum measurement. However, even if it is lower than the laser noise, the photodiode noise can be measured through the balanced photodiode experiment, in which a laser carrier (and noise) rejection factor of about $30 \mathrm{~dB}$ has been measured. The photodiodes noise appears to be $25 \mathrm{~dB}$ lower than the laser $1 / \mathrm{f}$ RIN, which demonstrates clearly that the main device to be optimized (or carefully selected) for low noise optical links at low frequency is the laser.
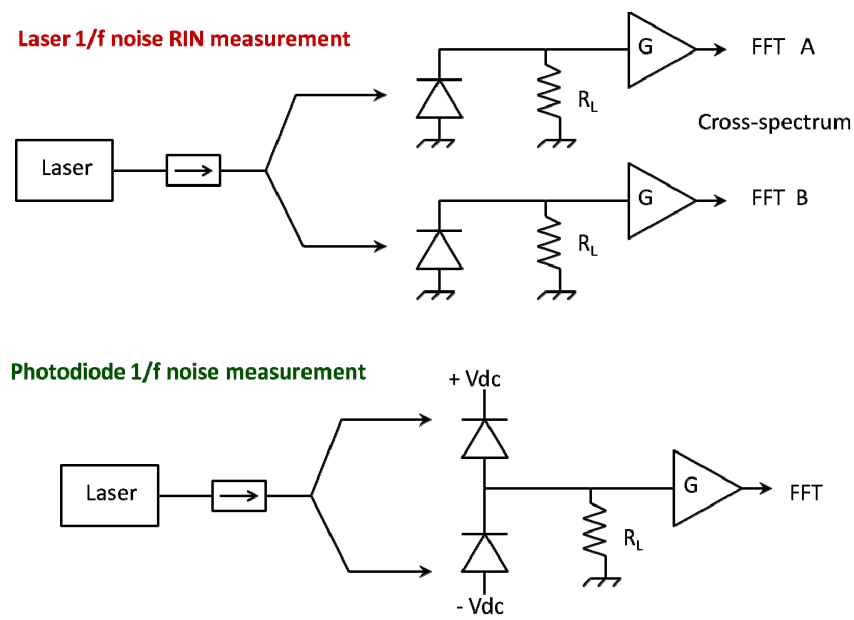

Figure 1 : The two measurement set-up used to characterize the laser amplitude noise and the photodiode noise at low frequencies $(\mathrm{f}<100 \mathrm{kHz})$

We can however take benefit of this balanced photodiodes set-up to investigate on the photodiodes noise versus the photocurrent. Up to our knowledge, the measurement of InGaAs photodiode 1/f noise under illumination has not been investigated up to now. Figure 3 presents the spectra obtained with different laser power levels and corresponding photodiode currents ranging from $4 \mathrm{~mA}$ to $8 \mathrm{~mA}$ (the effectively received power on each photodiode can be computed from the photodiode sensitivity, which is about $0.85 \mathrm{~A} / \mathrm{W})$. Also depicted are the shot noise levels computed from the photodiode current $\left(\mathrm{S}_{\text {shot }}=2 \mathrm{qI}\right)$ increased by $3 \mathrm{~dB}$ to take into account the two noise currents. This type of measurement is however difficult to perform, particularly when the photodiode current drops too much, which explains that only three current values are depicted here (which is not sufficient to get a current dependence for this $1 / \mathrm{f}$ noise).
However, these measurements clearly demonstrate that the photodiode $1 / \mathrm{f}$ noise is much lower than the laser $1 / \mathrm{f}$ noise, and should not be a problem in applications such as RF frequency references transmission or, even, interferometers realized at telecommunications wavelengths. Finally, the only problem with the photodiode lies in its nonlinear behavior.

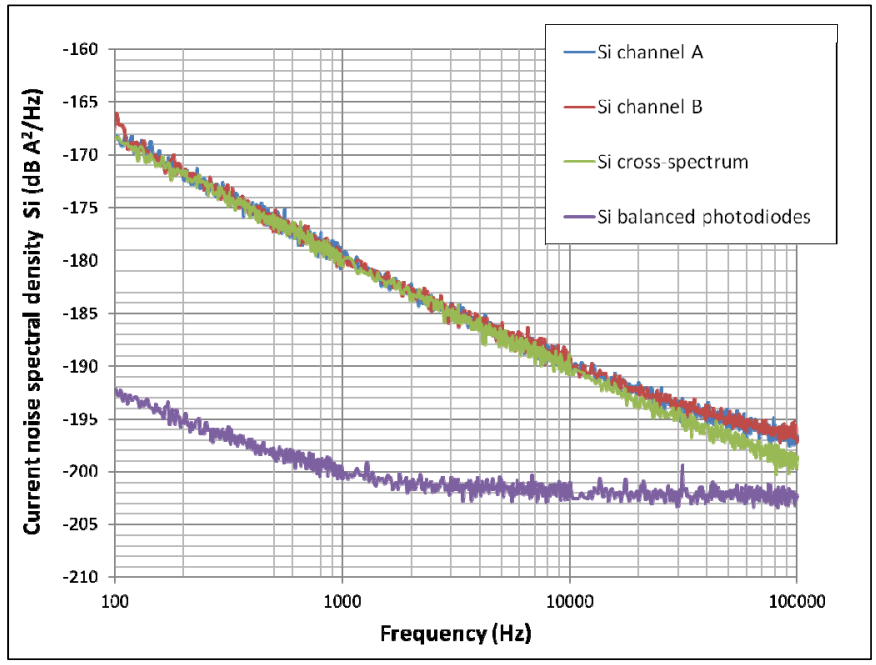

Figure 2 : Current noise on each channel and cross spectrum (laser noise) and current noise for balanced photodiodes (lower curve $=$ photodiode noise)

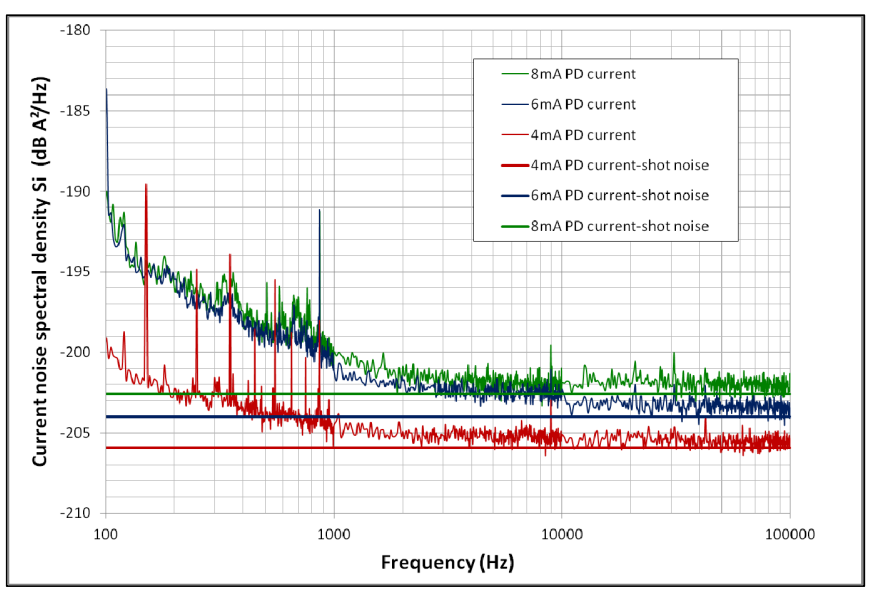

Figure 3 : Photodiodes current noise measured for three different photocurrent levels, and comparison with the shot noise level (the noise of a single photodiode is $3 \mathrm{~dB}$ lower than this measured noise)

Indeed, it has been shown $[3,8]$ that the photodiode can slightly shift the phase of an RF signal if the laser power is modified. This laser AM noise conversion into RF phase noise is one of the main problems in low phase noise optical links at microwave frequencies. Fortunately, the optical AM to RF phase conversion coefficient is largely dependent on the optical power received on the photodiode, and generally an optimum where this coefficient is close to zero can be found. If this optimized laser power is compatible with the foreseen application (if it is not too low, which would degrade the CNR), the optical link operation under this specific condition makes the RF link almost insensitive to photodiode parameters. 


\section{FIBER EXCESS NOISE}

Excess low frequency noise is not only observed in the optical link emitter and receiver. This type of noise can be directly generated in the optical fiber $[9,10,11]$, and particularly if the fiber length is large and the optical power inside the fiber is high. In the case of kilometer range fibers, three phenomena contribute to the excess low frequency noise at the output of the link, and also to the conversion of low frequency noise into RF noise in analog transmissions : the noise related to Rayleigh scattering in the fiber $[9,10,11]$, the noise related to the stimulated Brillouin scattering [9,10,11] and a possible conversion of laser FM noise through the fiber frequency dispersion [4].

Stimulated Brillouin scattering (SBS) is the starting up of a contra-propagative parasitic signal, shifted from the carrier of about $10.86 \mathrm{GHz}$ (standard SMF fiber), generated by an acousto-optic phenomenon inside the fiber. When the Brillouin oscillation has started, it mixes with the RF modulation signal and increases its phase and amplitude noises close to the carrier. The SBS threshold power can be evaluated thanks to this equation [7] :

$$
P_{t h}=\frac{21 K A_{e f f}}{g_{B} L_{e f f}}
$$

with $K$ being a fiber constant $\left(\mathrm{K}=1.5\right.$ for SMF fiber), $A_{\text {eff }}$ being the mode effective area, $L_{\text {eff }}$ being the effective length and $g_{B}$ the Brillouin gain $\left(5 \cdot 10^{-11} \mathrm{~m} / \mathrm{W}\right.$ in silica). According to this computation, the threshold will be close to $8.3 \mathrm{dBm}$ optical power at the input of a $10 \mathrm{~km}$ fiber, which is largely in the range of medium power telecommunications lasers. The two solutions to prevent from this noise are : using short enough links (or low optical power), or adding optical isolators every two or three kilometers in the link.

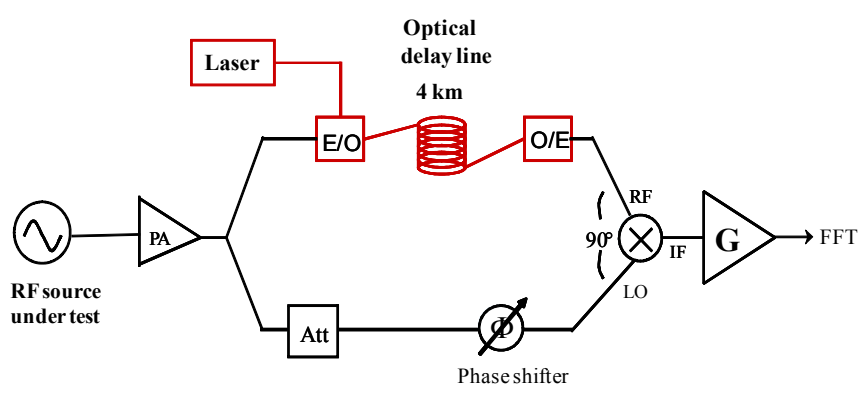

Figure 4 : Microwave frequency discriminator using a $4 \mathrm{~km}$ optical delay line

The conversion of laser FM noise into RF phase noise through the fiber dispersion parameters has been investigated in an optical delay line frequency discriminator [4], such the one depicted in Figure 4. This system is particularly useful for the measurement of low phase noise microwave sources. The microwave frequency noise is converted into phase fluctuations through the delay line, and then is demodulated thanks to a mixer acting as a phase detector. The interest of using optics in such a system lies in the possibility to use very large time delay, and the sensitivity of the system is directly proportional to this delay. However, this system can also be used for investigations on noise in optical fibers. In this case, an ultra high spectral purity microwave source feeds the frequency discriminator, and the observed noise at the output is the phase noise of the optical link. In the work presented in reference [4], we had carefully selected an optical spool of $4 \mathrm{~km}$ in order to stay lower than the SBS threshold, but other types of noise have been measured. The case of the conversion of laser FM noise through fiber dispersion is described in details in this paper, and we will not describe it here again. This problem can be solved by using a dispersion shifted fiber (DSF), which features a dispersion coefficient more than four times lower than the one of conventional SMF. However, even below the SBS threshold and using DSF fiber, excess LF noise was still observed in this experiment featuring spectral shapes very different from the one of the laser diode (which is almost pure $1 /$ f close to the carrier).

The origin of this excess low frequency noise (and the resulting RF phase noise) is related to the Rayleigh scattering, which is still occurring in optical fibers at relatively low optical power. A decrease of the optical power allowed us to improve the system performance close to the carrier, but this was also degrading the signal to noise ratio, and thus the far from carrier phase noise. It was thus difficult to get simultaneously a low phase noise floor close to the carrier $(1 \mathrm{~Hz}$ to $1 \mathrm{kHz})$ and far from the carrier ( $1 \mathrm{kHz}$ to $50 \mathrm{kHz}$ ).

A solution to this problem has been found by some researchers working on optical delay line oscillators, or OEOs (Optical Electric Oscillators). They found that the phase noise could be greatly improved if a low frequency modulation is added in the system $[3,10]$. A low modulation index sinusoidal perturbation is imposed on the laser current, at frequencies between $10 \mathrm{kHz}$ and $100 \mathrm{kHz}$, thus modulating the laser frequency. The result of this small frequency spreading of the optical signal is that it becomes less sensitive to interference or polarization fluctuations.

For low noise systems design, the problem with such a technique is that the modulation signal is often in the measurement frequency range. However, in the case of our frequency discriminator, a $100 \mathrm{kHz}$ modulation frequency has been chosen, and this frequency is above the $50 \mathrm{kHz}$ bandwidth of the experiment (the fiber delay involves a loss of sensitivity at every frequency $f_{\text {offset }}$ where $\pi \tau f_{\text {offset }}$ is equal to an integer multiple of $\pi$, which means a limit in the measurement frequency range equal to $1 / \tau$; for $4 \mathrm{~km}$ fiber spool, $\tau=18.9 \mu \mathrm{s}$ and thus $\mathrm{f}_{\max }=50 \mathrm{kHz}$ ).

The experiment has thus been performed with a medium power laser (EM4, $60 \mathrm{~mW}$ laser module) directly modulated on its current by a $100 \mathrm{kHz}$ sinusoidal signal. This optical source feeds a Mach Zehnder modulator and then a $4 \mathrm{~km}$ SMF spool, with an optical isolator at its input. After being delayed, the signal ends on a fast photodiode (Discovery DSC30S), and then is compared to the microwave reference source thanks to a low $1 /$ f noise mixer (silicon shottky diodes mixer).

In order to evaluate the discriminator noise floor, an ultra stable microwave frequency source is used. This source is a sapphire oscillator, featuring a phase noise lower than $-135 \mathrm{dBc} / \mathrm{Hz}$ at $1 \mathrm{kHz}$ offset from a $4.85 \mathrm{GHz}$ oscillating frequency [12], which is still a better performance than the one 
which can be obtained with the optical delay line discriminator (at least for a single spool and single mixer discriminator, with no correlation system involved). The result of the measurement of the sapphire source is depicted in Figure 5.

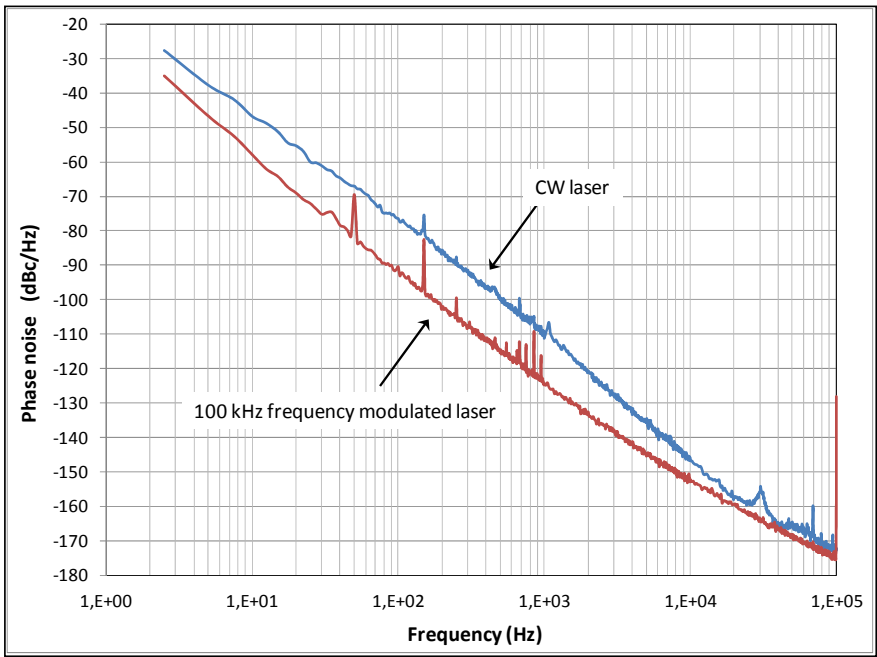

Figure 5 : Noise floor of the optical delay line microwave frequency discriminator, measured at $4.85 \mathrm{GHz}$ using a sapphire oscillator

It is clear on this Figure that the noise floor is highly sensitive to the $100 \mathrm{kHz}$ modulation of the laser current. If this system is not used, a bump is observed on the spectrum, very similar to the shape of a generation-recombination noise. This is probably due to the dynamics of the Rayleigh scattering process, which increases the phase noise floor of more than $10 \mathrm{~dB}$ between $10 \mathrm{~Hz}$ and $1 \mathrm{kHz}$. When the modulation signal is on, the phase noise spectrum features again a $30 \mathrm{~dB} /$ decade slope at these frequency offsets, which is the signature of an almost pure $1 /$ f noise.

\section{CONCLUSION}

A measurement technique to characterize the photodiodes 1/f noise under illumination (photocurrent noise) has been proposed. The 1/f noise level observed in InGaAs photodiodes is very low, and should be masked by the laser $1 / \mathrm{f}$ noise in most applications. However, the measurement technique can be used to investigate more deeply on the physical mechanisms involved in photogeneration.

Apart from the optoelectronic devices, laser and photodiode, the fiber spool has been found to be a strong contributor to $1 / \mathrm{f}$ noise and close to the carrier RF phase noise. Various scattering processes in the fiber are responsible for the generation of this excess noise, and different techniques have been described to reduce this type of noise.

\section{REFERENCES}

[1] B. Onillon, B. Benazet, O. Llopis, “Advanced Microwave Optical Links for LO Distribution in Satellite Payloads" IEEE International Topical Meeting on Microwave Photonics, MWP 2006, Grenoble, Oct. 2006.

[2] X.S. Yao, D. Eliyahu, L. Maleki, "Progress in the optoelectronic oscillator - a ten year anniversary review," IEEE Microwave Theory and Tech. Symp. Digest, vol. 1, pp. 287-290, Jun. 2004.

[3] D. Eliyahu, D. Seidel, L. Maleki, "RF Amplitude and Phase-Noise Reduction of an Optical Link and an Opto-Electronic Oscillator", IEEE Trans. on Microwave Theory and Tech., Vol. 56, N. 2, Feb. 2008.

[4] H. Brahimi, P. Lacroix, O. Llopis, "Optimisation of a microwave frequency discriminator based on an optical delay line", IEEE Int. Topical Meeting on Microwave Photonics, MWP 2009, Valencia, Oct. 2009.

[5] H. Brahimi, H.L. Martinez-Reyes, P.H. Merrer, A. Bouchier, O. Llopis, "A CAD approach of microwave optical systems including noise performance", Proc. of the 2009 European Microwave Conf., Rome, oct. 2009, pp. 1642-1645.

[6] E. Rubiola, E. Salik, N. Yu, L. Maleki, "Flicker noise in high speed photodiodes", IEEE Trans. on Microwave Theory and Tech., vol 54, $\mathrm{n}^{\circ} 2$, Feb 2006, pp. 816-820.

[7] G.P. Agrawal, "Fiber-optic communication systems" Wiley Ed., 1997.

[8] K. Saleh, P.H. Merrer, O. Llopis, G. Cibiel, "Optoelectronic Oscillator Based on Fiber Ring Resonator: Overall System Optimization and Phase Noise Reduction”, IEEE-Int. Frequency control Symposium, Baltimore, May 2012.

[9] O. Okusaga, J. Cahill, W. Zhou, A. Docherty, G.M. Carter, C.R. Menyuk, "Optical scattering induced noise in RF-photonic systems" IEEE-Int. Frequency control Symposium and EFTF, San Francisco, May 2011.

[10] O. Okusaga, W. Zhou, J. CAhill, A. Docherty, C.R. Menyuk, "Fiber induced degradation in RF over fiber links", IEEE-Int. Frequency control Symposium, Baltimore, May 2012.

[11] K. Saleh, O. Llopis, G. Cibiel, "Optical Scattering Induced Noise in Fiber Ring Resonators and Optoelectronic Oscillators", Journal of Lightwave Technology, vol. 31, no. 9, pp. 1433,1446, May 1, 2013.

[12] G. Cibiel, M. Regis, O. Llopis, A. Rennane, L. Bary, R. Plana, Y. Kersale, V. Giordano "Optimisation of an ultra low phase noise sapphire - SiGe HBT oscillator using nonlinear CAD” IEEE trans. on Ultrasonics Ferroelect. and Freq. Control, Vol. 51, n ${ }^{\circ}$ 1, Jan. 2004, pp. 33-41. 